\section{Scrotal swelling in sarcoidosis}

Shahid A Khan MRCS Jonathan L Richenberg FRCR Mathew S Fletcher FRCS

J R Soc Med 2004;97:487-488

SECTION OF UROLOGY, 26 FEBRUARY 2004

Ultrasound is central to the investigation of a scrotal mass, determining whether or not the testes are involved and the nature of any lesions. In a patient with evidence of sarcoidosis elsewhere, surgical intervention may be avoidable.

\section{CASE HISTORY}

For two months an Afro-Caribbean man aged 35 had been aware of painless scrotal swellings, an enlarging submental mass and multiple small cutaneous lumps on his arms and trunk. He also reported weight loss, lethargy and night sweats. On examination he had bilateral hydroceles, cervical lymphadenopathy, a large non-tender submental swelling and numerous firm cutaneous lumps. Laboratory investigations revealed eosinophilia and a raised erythrocyte sedimentation rate but normal testicular tumour markers. On scrotal ultrasound, in addition to the hydroceles there were geographical echo-poor areas within both testes suggesting a diffuse infiltrate (Figure 1). Bilateral symmetrical hilar lymphadenopathy was noted on the chest radiograph while nothing abnormal was seen on CT of the abdomen and pelvis. Serum angiotensin-1-converting enzyme level was raised at $180 \mathrm{IU} / \mathrm{L}$ (normal range 1236) and pulmonary function tests showed a restrictive defect. A fine-needle aspirate from the submental mass and excision biopsies from a lesion on the right arm were consistent with sarcoidosis. Tests for vasculitis, syphilis, Epstein-Barr virus, acid-fast bacilli and fungi were all negative.

Neither testicular biopsy nor an orchidectomy was deemed appropriate. The subsequent clinical course was consistent with the diagnosis of sarcoidosis. With steroid therapy the hydroceles disappeared and the ultrasonic features improved (Figure 2).

Departments of Urology and Radiology, Royal Sussex County Hospital, Eastern Road, Brighton BN2 5ES

Correspondence to: Shahid A Khan, Department of Urology, Sussex House, 1 Abbey Road, Brighton BN2 1ES, UK

E-mail: saak_2000@yahoo.co.uk

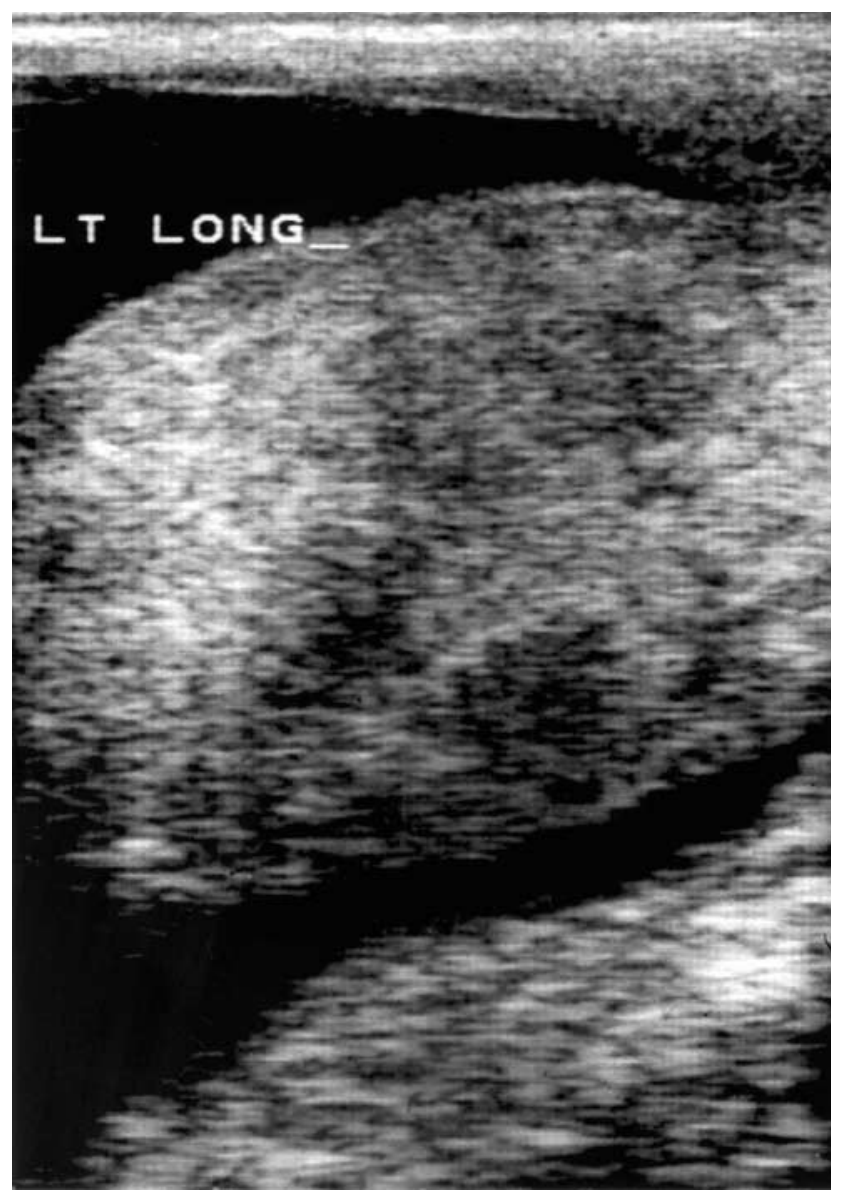

Figure 1 Ultrasound left scrotum (7.5 MHz linear array)

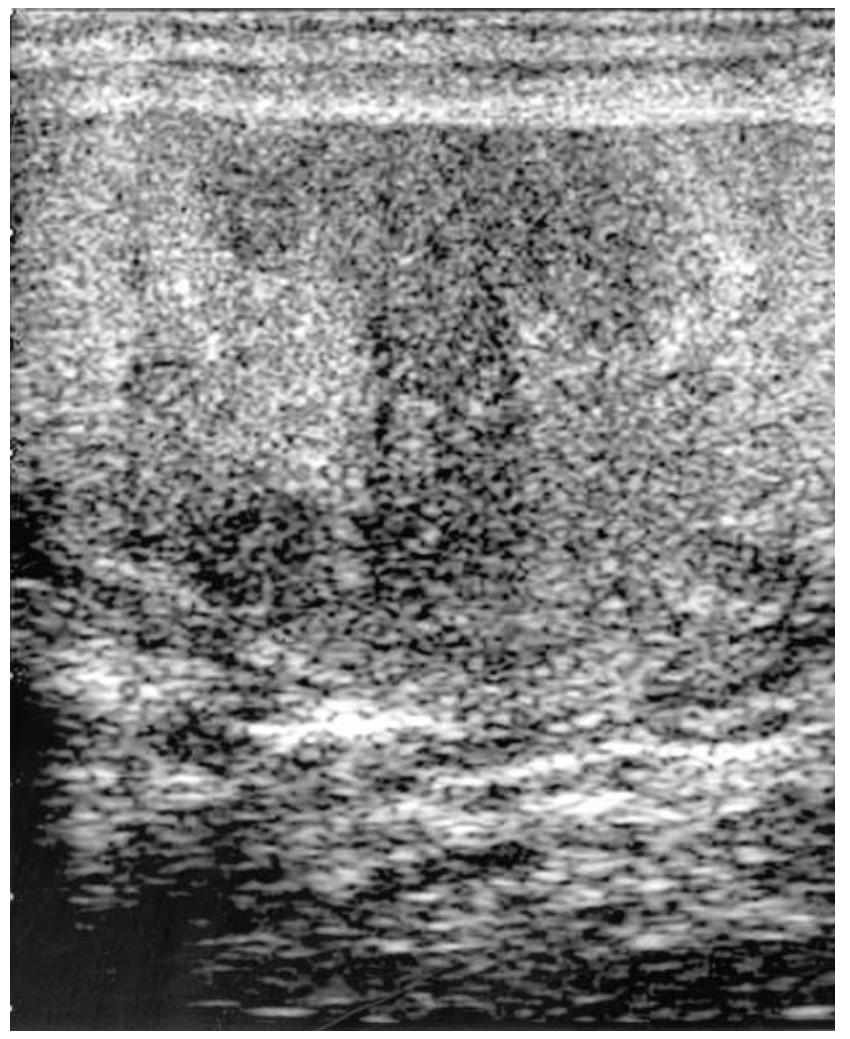

Figure 2 Ultrasound left scrotum at 2-year follow-up (highfrequency $10 \mathrm{MHz}$ linear array) 


\section{COMMENT}

Sarcoidosis is a systemic disease of unknown aetiology that results in the formation of non-caseating granulomas in multiple organs. The prevalence is higher among blacks than whites by a ratio of $20: 1$. Usually the disease is localized to the chest, but urogenital involvement is found in $0.2 \%$ of clinically diagnosed cases and 5\% of those diagnosed at necropsy. ${ }^{1,2}$ The kidney is the most frequently affected urogenital organ, followed in men by the epididymis. Testicular sarcoidosis can present as a diffuse painless scrotal mass or can mimic acute epididymo-orchitis. Usually it appears with systemic manifestations of the disease. ${ }^{3}$ Since it causes occlusion and fibrosis of the ductus epididymis, fertility may be affected. ${ }^{4}$ On ultrasound, the hypoechogenicity and 'infiltrative' pattern seen in the present case are recognized features. ${ }^{5}$ Opinions differ on the need for histological proof, with reports of limited biopsy and frozen section, radical orchidectomy in unilateral disease and unilateral orchidectomy in bilateral disease. The peak incidence of sarcoidosis and testicular neoplasia coincide at 20-40 years and this is why most patients end up having an orchidectomy. However, testicular tumours are much more common in white men, less than $3.5 \%$ of all testicular tumours being found in black men. ${ }^{6}$ These racial variations justify a more conservative approach in patients of AfroCaribbean descent with proven sarcoidosis elsewhere. Careful follow-up and ultrasonic surveillance may be preferable in certain clinical settings to biopsy and surgery-especially in patients with bilateral testicular disease.

\section{REFERENCES}

1 Turk CO, Schacht M, Ross L. Diagnosis and management of testicular sarcoidosis. J Urol 1986;135:380-1

2 Gross AJ, Heinzer H, Loy V, Dieckmann KP. Unusual differential diagnosis of testis tumour: intrascrotal sarcoidosis. J Urol 1992;147: 111-14

3 Geller RA, Kuremsky DA, Copeland JS, Stept R. Sarcoidosis and testicular neoplasm: an unusual association. J Urol 1977;118:487-8

4 Svetec D, Waguespack RL, Sabanegh ES Jr. Intermittent azoospermia associated with epididymal sarcoidosis. Fertil Steril 1998;70:777-9

5 Eraso CE, Vrachliotis TG, Cunningham JJ. Sonographic findings in testicular sarcoidosis simulating malignant nodule. J Clin Ultrasound 1999;27:81-3

6 Daniels JL Jr, Stutzman RE, McLeod DG. A comparison of testicular tumors in black and white patients. J Urol 1981;125:341-2 